\title{
The Only Probable Way to Understand Universe
}

\author{
Jivesh Adhlakha
}

June 02,2020

\section{Abstract}

In this paper, I have deduced through logical and mathematical arguments that there is only one way to approach physical phenomena in order to understand the correct picture of physical world. Further, it has been deduced that this approach requires all phenomena to be explained in an emergent framework with one and only one underlying principal. Hence, it directly paves the way to a single theory that can explain all the phenomena of the Universe with same underlying reasoning - both at microscopic and macroscopic scales. Therefore, a probable approach to Unified Theory is asserted. 


\section{Solution to the Problem Presented by The Dilemma}

\section{Theorem of Scientific Models}

\section{In the preprint proposing The Dilemma Theorem of Scientific Models}

(Adhlakha, 2020), it was deduced that the model based approach of science fails to discover and assert the actual working of physical world. I would like to propose a solution to it by initially considering the very example of $\mathbf{4} \underline{\text { (see, example) }}$ used in that preprint. The problem was that the same observation 4 had multiple equally probable and successful explanatory models, making it impossible to assert with confidence how does 4 actually happens in the Universe - By multiplication of two 2s or addition? However (if we look at it), if we keep on trying to explain that 4 using twos, it will not solve our problem. Only if we try to find the very basics of 4 - how does it actually happen, and why is $\mathbf{4}$ actually even there - we will find an explanation which tells what actually happens rather than becoming a probable model based explanation. If we go to the basics, we find that it is not actually $2 \mathrm{~s}$ which make 4 but even more fundamental 1 s are responsible for both 4 and 2 , which give way to 4 . Further, these 1 s explain not only 4 s but every possible phenomena (referring to whole system of natural numbers representing different 
phenomena in this example) using a single underlying process - Addition. We find that multiplication of $1 \mathrm{~s}$ fails to explain any phenomena in this approach from very roots of all physical world (which otherwise was an equally probable scenario for separate processes). This leaves only one possibility - all the phenomena (including $4 \mathrm{~s}$ and $2 \mathrm{~s}$ ) happen due to 1 and its addition. All the other processes and observations stem from this one underlying element and only one process being applied on it (addition on $\mathbf{1 s ) . ~ T h i s ~ a p p r o a c h , ~ w h i c h ~ e x p l a i n s ~ a l l ~ t h e ~ p h e n o m e n a ~}$ through one underlying quantity and its dynamics, solves the problem presented by dilemma theorem of scientific models and rules out all other possibilities, which were otherwise equally successfully explaining different phenomena in their individual domains.

Extending this to domain of physical sciences, the only way to understand and assert the actual understanding of Physical World is that every single phenomenon emerges through a single quantity and process at play at its root. And as seen in the case discussed above, there can be only one such possible scenario (addition on $1 \mathrm{~s}$ in the case discussed). So, if such a theory is found, we can be sure of that's how physical world actually works because there can only be one such case where everything is explained in an emergent framework, that too through a single principal at work. Based upon this, I propose a Principal - The Emergent

\section{Principal of Physicality.}




\section{The Emergent Principal of Physicality}

If we try to find separate model based explanations for different processes and try to link them, not only it might not be possible at all, but as the Dilemma Theorem tells, it is impossible to assert that found explanation is how a phenomenon actually happens. Therefore, the only way to solve this dilemma is by looking at the common root of all the phenomena. This is because there can be no alternative to an explanation that describes everything in Physical World - both microscopic and macroscopic - through a single principal and everything emerges from it. Therefore, there can only be one such theory which explains everything about physical world in an emergent manner and that too with single principal at its heart. Hence, it does not just remain a theory but the very way the physical Universe works. This indicates that everything in Universe emerges through a single principal at work, presenting itself in various possibilities. I call this - The Emergent Principal of Physicality. To put it neatly:

The Emergent Principal of Physicality states that every phenomenon in Physical World happens due to a single principal at work, presenting itself in various possible scenarios in an emergent manner. 
This tells us to make a shift in our approach towards understanding physical world and look for the principal that is at work to explain everything in an emergent manner. Therefore, only an Emergent Theory of Everything with one principal at root will explain how the Physical world actually works. I call such a theory - Uniprincipal Emergent Theory of Everything (Uniprincipal ETE). All other approaches are subject to the problem proposed by dilemma theorem of scientific models and therefore, make it impossible to find and assert understanding of physical world.

\section{Summary}

The Dilemma Theorem of Scientific Model shows that it is impossible for Model based approach of Physical Sciences to actually explain physical world. Therefore, only an Emergent Theory of Everything based upon a single principal (Uniprincipal ETE) can tell us how physical world works. This is because, there can only be one such possibility which explain the root of all physical phenomena in an emergent manner through single principal. Also because, there can be only one such principal, which when discovered, will help us to explain Physical World. 


\section{Conclusion}

The Dilemma Theorem of Scientific Models and The Emergent Principal of Physicality combined, tell that it is impossible for Physical Sciences to understand physical world through model based approach. The only way to find actual explanation to working of Physical world is through Uniprincipal Emergent Theory of Everything. Therefore, Physical Sciences need to change their approach and look for a single principal that explains physical world. Further, the Emergent Principal of Physicality has a lot of consequences for current sciences as well as possible future. It also opens the door to speculate all the aspects of Physical World and the way we have seen it. All this shall be discussed in separate paper. 


\section{References and Citations}

1) Adhlakha, J. (2020, May 20). The Inherent Limitation of Model Based Approach of Physical Sciences to Understand Universe. https://doi.org/10.31219/osf.io/r85p2 